\title{
Solitary non-parasitic liver cyst treated by cyst-gastrostomy
}

\author{
D. Chang
}

Professorial Surgical Unit, Westminster Hospital, London SWIP 2AP, UK.

\begin{abstract}
Summary: A 66 year old female presented with a symptomatic solitary non-parasitic liver cyst. This was treated by a cyst-gastrostomy following which the patient has remained well without complications for 12 years. Given an appropriate anatomical relationship of cyst and stomach, this would appear to be a safe and effective operation.
\end{abstract}

\section{Introduction}

Hepatic cysts can be divided into parasitic and nonparasitic. The majority of patients with nonparasitic liver cysts are found to have these cysts incidentally at laparotomy or on radiological investigation of the upper abdomen. Symptomatic cases are uncommon ${ }^{1,2}$ but those that are require operative treatment. The options for these patients include enucleation, partial hepatic resection, wide unroofing, incision and external drainage or internal drainage into a jejunal loop. This case report is of the first successful cyst-gastrostomy, with drainage of a massive solitary non-parasitic hepatic cyst into the stomach, with follow-up for 12 years.

\section{Case report}

A 66 year old woman, who had emigrated from South Africa 15 years previously, presented in 1972 with a 3-year history of epigastric pain aggravated by posture. She had noticed her abdomen had become distended over this period.

Examination revealed a tense mass in the epigastrium extending from both costal margins to below the umbilicus (Figure 1). General examination was otherwise normal. A barium meal showed displacement of the stomach and duodenum downwards whilst a liver isotope scan revealed both hepatic lobes displaced up and laterally. Liver function tests were normal and a hydatid complement fixation test was negative.

In view of her symptoms, a laparotomy was carried out. A $20 \mathrm{~cm}$ thin walled cyst was found

Correspondence: D. Chang, F.R.C.S., Department of Surgery, Royal Liverpool Hospital, Prescot Street, Liverpool L7 8XP, UK.

Accepted: 6 January 1988

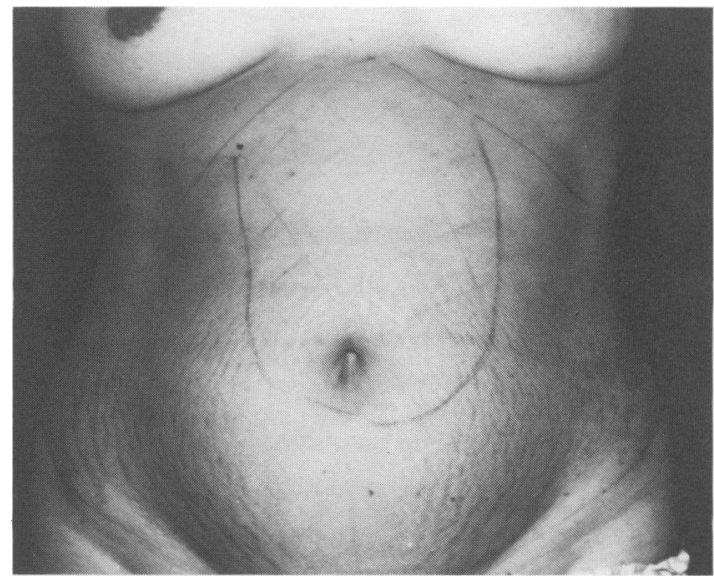

Figure 1 Patient with abdominal mass outlined.

which occupied the inferior part of the liver and projected into the falciform ligament (Figure 2). The cyst was decapped using the diathermy. It contained 2 litres of green fluid. A small bile duct opening within the cyst was under-run with linen sutures (Figure 3). The rest of the laparotomy was normal. Microscopy revealed a subcapsular cyst lined by columnar epithelium of bile duct origin. Post-operatively there were no complications.

She was seen again in 1975 with a 3-month history of right subcostal pain, radiating to her back. She had also noticed a swelling below the right costal margin. A mass occupying the right hypochondrium and epigastrium was found on this occasion. Radiological investigations showed a large mass arising from the liver displacing the transverse colon and hepatic flexure. Liver function tests were again normal. A second laparotomy in

(C) The Fellowship of Postgraduate Medicine, 1988 


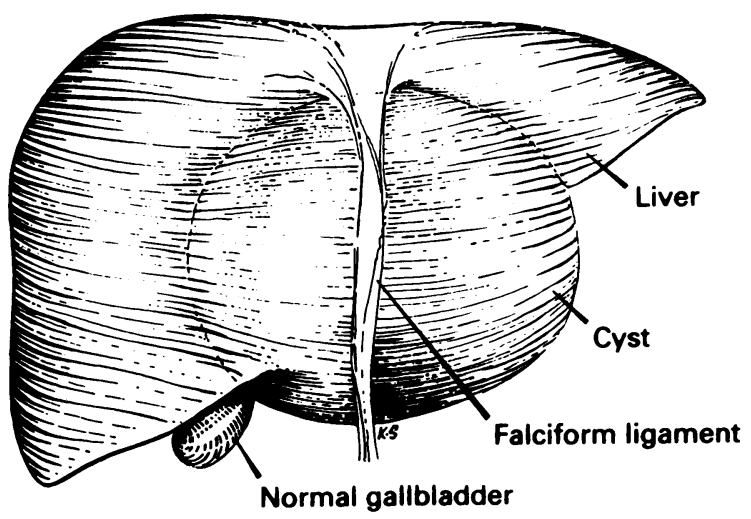

Figure 2 Anatomy at initial laparotomy.

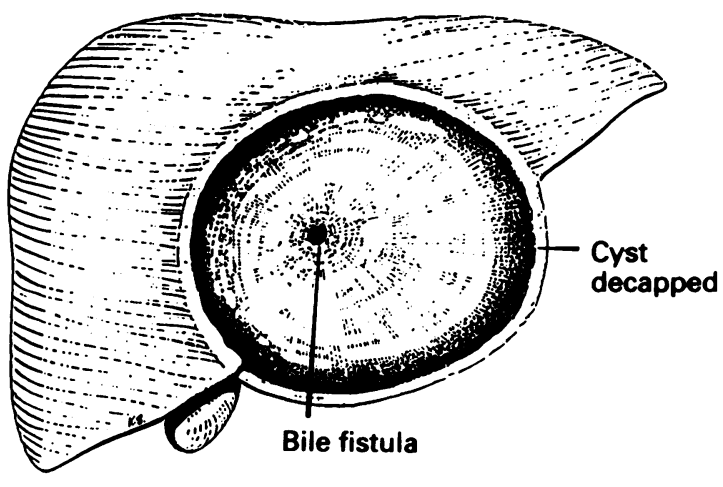

Figure 3 Liver cyst opened and decapped.

August 1975 revealed a massive lobulated cyst of the liver with the lesser curve of the stomach stretched over the cyst. The stomach was opened via an anterior gastrotomy and the cyst approached through the posterior wall of the stomach: 2 litres of greyish fluid were drained. The stomach wall and the cyst were opened for $4 \mathrm{~cm}$ and the edges sutured together with continuous catgut (Figure 4). A 2 layer repair of the anterior gastrotomy was performed. Histology showed recurrent cyst wall.

Post-operatively, she had a gastroscopy to check the patency of the cyst-gastrostomy. The opening was found to be widely patent though oedematous and friable. A follow-up barium meal 2 months later showed that contrast easily entered the cyst cavity.

During regular follow-up, she was seen again in February 1987 with upper abdominal pain and nausea. A large calculus in the gallbladder was seen on ultrasound scan but no hepatic cysts. A barium meal showed a small empty diverticulum related to
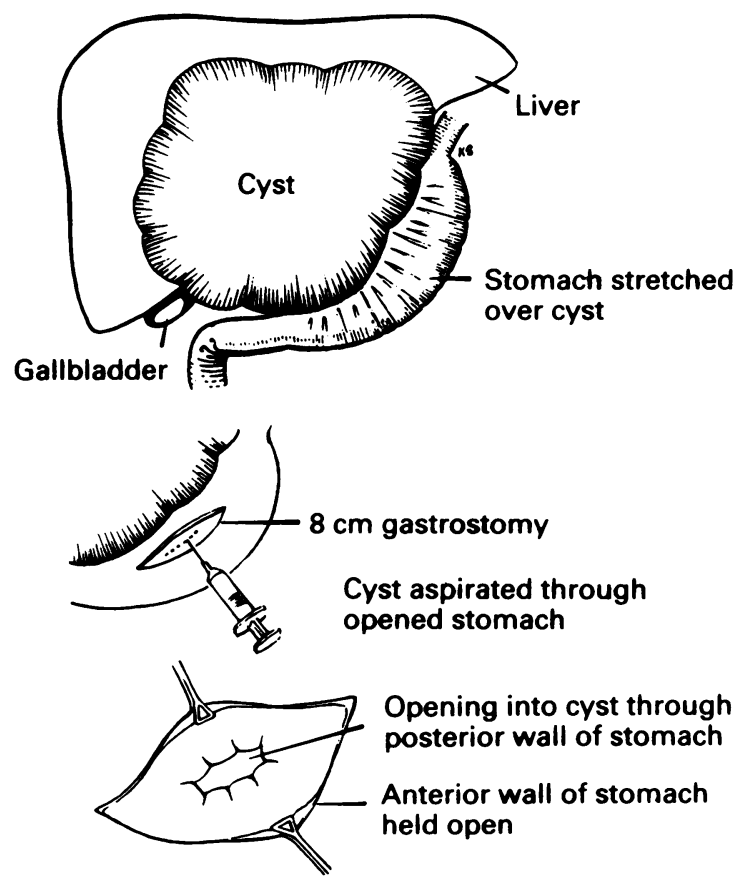

Figure 4 Steps during the cyst-gastrostomy.

the antrum of the stomach. This was probably the site of the original liver cyst. She subsequently had a cholecystectomy in May 1987. At operation it was noted that the stomach remained adherent to the liver. There was no evidence of recurrence of the cyst.

\section{Discussion}

The exact incidence of solitary non-parasitic liver cysts is difficult to estimate but would appear to be $0.1-0.2 \%$ of all autopsies. ${ }^{3,4}$ However, with the advent of ultrasound scanning in the investigation of upper abdominal disease such cysts are increasingly recognized.

The majority of non-parasitic cysts are asymptomatic ${ }^{1}$ but symptoms can arise usually as a result of pressure on the liver and adjacent organs or from complications. The commonest presentation is abdominal pain and an abdominal mass or fullness. $^{1,5}$ An asymptomatic non-parasitic liver cyst requires no treatment so long as parasitic cyst, tumour or abscess can be excluded.2,5

Various operative treatments have been advocated for the symptomatic patient. Many surgeons consider complete excision by enucleation or by hepatic resection to be the treatment of choice. $^{1,3,4,6,7}$ However, this latter method is 
hazardous with significant morbidity and mortality, unless the cyst is superficial or pedunculated.

Partial excision or simple incision and drainage is easily performed but carries a high incidence of cyst recurrence. ${ }^{2,4,5}$ Deroofing of the cyst with oversewing of ductal connections has been shown to be a highly effective and safe procedure. ${ }^{5}$ However, the cyst is still liable to recur, as illustrated in this case. External drainage with a tube cystostomy is usually performed for infected cysts. Internal drainage into a Roux-en-Y limb of jejunum is recommended when the cyst contents are bile stained, ${ }^{2,6}$ although sepsis may complicate this procedure. $^{6}$

\section{References}

1. Jones, W.L., Mountain, J.C. \& Warren, K.W. Symptomatic non-parasitic cysts of the liver. Br J Surg 1974, 61: 118-123.

2. Rashed, A., May, R.E. \& Williamson, R.C.N. The management of large congenital liver cysts. Postgrad Med J 1982; 58: 536-540.

3. Sanfelippo, P.M., Bearhs, O.H. \& Weiland, L.H. Cystic disease of the liver. Ann Surg 1974, 179: 922-925.

4. Williamson, R.C.N., Ramus, N.I. \& Shorey, B.A. Congenital solitary cysts of the liver and spleen. $\mathrm{Br} J$ Surg 1978, 65: 871-876.
This case illustrates the successful treatment of a recurrent solitary non-parasitic hepatic cyst by a cyst-gastrostomy. She has been followed up for 12 years so far without any evidence of complications. The relative sterility of a healthy stomach $^{8}$ may prevent septic complications seen with a jejunal drainage. ${ }^{5}$ If the anatomy of the cyst allows the approximation of cyst and stomach this would appear to be a safe and effective operation.

\section{Acknowledgement}

I wish to thank Professor Harold Ellis for permission to report this case and for his assistance in preparing this paper.

5. Litwin, D.E.M., Taylor, B.R., Greig, P. \& Langer, B. Non-parasitic cysts of the liver - the case for conservative surgical management. Ann Surg 1987, 205: 45-48.

6. Longmire, W.P., Mandiola, S.A. \& Gordon, H.E. Congenital cyst disease of the liver and biliary system. Ann Surg 1971, 174: 711-726.

7. Russell, R.C.G. Ruptured solitary cysts of the liver. $\mathrm{Br}$ J Surg 1972, 59: 919-920.

8. Gorbach, S.L. Intestinal microflora. Gastroenterology 1971, 60: 1110-1129. 